\title{
Detection of viral infections in wild Korean raccoon dogs (Nyctereutes procyonoides koreensis)
}

\author{
Dong-Kun Yang ${ }^{1, *}$, Seoug Heon Lee ${ }^{1}$, Ha-Hyun Kim ${ }^{1}$, Jong-Taek Kim², Sangin Ahn², In-Soo Cho ${ }^{1}$ \\ ${ }^{1}$ Animal and Plant Quarantine Agency, Ministry of Agriculture, Food and Rural Affair, Gimcheon 39660, Korea \\ ${ }^{2}$ College of Veterinary Medicine, Kangwon National University, Chuncheon 24341, Korea
}

(Received: October 9, 2017; Revised: November 17, 2017; Accepted: December 4, 2017)

\begin{abstract}
Wild raccoon dogs (Nyctereutes procyonoides koreensis) may play a role transmitting several pathogens to humans and pet animals. Information concerning the incidence of rabies, canine distemper virus (CDV), canine parvovirus (CPV), canine adenovirus type 2 (CAdV-2), canine parainfluenza virus type 5 (CPIV-5), and canine herpesvirus (CHV) is needed in wild raccoon dogs. In total, 62 brain samples of raccoon dogs were examined for rabies virus (RABV) and CDV, and 49 lung samples were screened for CDV, CAdV-2, CPIV-5, and CHV. No RABV, CAdV-2, CPIV-5, or CHV was identified, but nine CDV antigens $(8.1 \%, 9 / 111)$ were detected. Moreover, 174 serum samples from wild raccoon dogs were screened for antibodies against the five major viral pathogens. The overall serosurveillance against CDV, CPV, CAdV-2, CPIV-5, and CHV in wild raccoon dogs was $60.3 \%, 52.9 \%, 59.8 \%, 23.6 \%$, and $10.3 \%$, respectively. Comparisons of the sero-surveillance of the five pathogens showed that raccoon dogs of Gyeonggi province have slightly higher sero-positive rates against CDV, CPV, and CHV than those of Gangwon province. These results indicate high incidences of CDV, CPV, and CAdV-2 in wild raccoon dogs of two Korean provinces and a latent risk of pathogen transmission to companion and domestic animals.
\end{abstract}

Keywords: canine distemper, rabies, raccoon dogs, sero-surveillance

\section{Introduction}

Wild raccoon dogs (Nyctereutes procyonoides koreensis) are becoming more likely to invade human residences due to shortages of food during winter, and they may even live in human habitats because of easy access to food. Frequent encounters between wild raccoon dogs and humans increase the incidence of zoonosis. In particular, the raccoon dog is known to be responsible for transmitting many pathogens, including rabies virus (RABV) and Demodex canis in Europe and Asia [4, 18]. A "One Health" concept has been established to prevent the spillover of zoonotic infectious diseases [9]. Contact between raccoon dogs and companion animals can also increase the incidence of infectious diseases such as canine distemper virus (CDV) and kennel cough in dogs and cats.

Rabies is caused by RABV belonging to the genus Lissavirus, family Rabdoviridae, and is divided into human and animal rabies. Animal rabies cases have been identified in a variety of animals in the Republic of Korea, including cattle, dogs, raccoon dogs, and cats [21]. According to an epidemiological study of rabies in Korea, all animal rabies cases have been linked to rabid raccoon dogs since 1993. Therefore, raccoon dogs have been attracting attention as a carrier that transmits rabies. Canine distemper caused by CDV belonging to the genus Morbillivirus, family Paramyxoviridae, leads to lethal disease in many animal species, including raccoon dogs. Only a single serotype against CDV is known to exist. Wild Korean raccoon dogs infected with CDV have been reported using a commercial CDV antigen detection kit $[4,6]$. Canine parvovirus (CPV) belonging to the genus Parvovirus, family Parvoviridae, causes gastrointestinal illness (e.g., acute hemorrhagic diarrhea) in young puppies [7]. Animals infected with CPV excrete large amounts of virus in their feces after a natural infection, which is transmitted to CPV antibody-negative puppies. The incidence of CPV in raccoon dogs has been reported in Germany and Japan [8]. Moreover, recent advances in molecular technology such as the next generation sequencing, polymerase chain reaction (PCR), and a variety of enzyme-linked immunosorbent assay (ELISA) kits have enabled the detection of many infectious diseases in wild raccoon dogs. Based on advanced techniques, the presence of several infectious diseases, including canine adenovirus type $2(\mathrm{CAdV}-2)$, canine parainfluenza virus type $5(\mathrm{CPIV}-5)$, and canine herpesvirus type $1(\mathrm{CHV})$, representing a problem in raccoon dogs and pet dogs, have been reported in Italy and Norway [3, 13].

*Corresponding author

Tel: +82-54-912-0785, Fax: +82-54-912-0812

E-mail: yangdk@korea.kr 
Among infectious pathogens, rabies causes the most catastrophic damage in all warm-blooded animals. It is necessary to identify pathogens in wild raccoon dogs residing in rabies-risk provinces and to continuously monitor major pathogens circulating in wild raccoon dogs to prevent rabies and many other infectious diseases. In this study, we investigated the incidence rates of RABV, CDV, CAdV-2, CPIV-5, and $\mathrm{CHV}$ from brain or lung samples of raccoon dogs and determined the sero-surveillance of CDV, CPV, CAdV-2, CPIV-5, and CHV in wild Korean raccoon dog sera.

\section{Materials and Methods}

\section{Sample collection}

In total, 62 brain and 49 lung samples were obtained from the Wild Animal Rescue Center of Kangwon National University to determine incidence rates. For sero-surveillance, blood samples were obtained from 174 wild raccoon dogs as part of annual surveillance for the national rabies eradication program. Blood was taken from the cephalic vein. In total, 71 and 103 serum samples were obtained from raccoon dogs living in Gyeonggi and Gangwon Provinces, respectively from February 2013 to June 2017. Clotted blood samples were centrifuged $(3,000 \times \mathrm{g}, 10 \mathrm{~min})$, and the sera were stored at $-20^{\circ} \mathrm{C}$ until use.

\section{Detection of viral pathogens}

A direct fluorescent antibody (DFA) test was carried out to detect RABV in 62 brain samples using the procedure described by OIE [20]. Briefly, thin, frozen sections of raccoon dog brain were placed on a slide and fixed with cold acetone $\left(-20^{\circ} \mathrm{C}\right)$ for $20 \mathrm{~min}$. The slides with fixed brain tissue were subsequently reacted with a specific monoclonal antibody (MEDIAN Diagnostics, Korea) against RABV for $1 \mathrm{~h}$. After washing with phosphate-buffered saline (PBS), the slides were stained with fluorescent isothiocyanate-conjugated goat-anti mouse IgG+IgM (MEDIAN Diagnostics). Samples showing specific fluorescence in brain cells were diagnosed as positive. Commercial RT-PCR kits (Canine distemper virus detection kit and Canine parainfluenza virus detection kit) manufactured by iNtRON Biotechnology (Korea) were used to detect CDV and CPIV-5 antigens in brain and lung samples according to the manufacturer's instructions. Primer sets (forward: 5'-TCCCGTTCACCAGCACCAGGGCC-3', reverse: 5'-GGTGAGAGGCGGGGGAGGGGT3') specific for the CAdV-2 gene were prepared and mixed with PCR premix (Bioneer, Korea) and the extracted lung sample DNA. In brief, PCR was carried out in a PCR premix containing $10 \mu \mathrm{L}$ of denatured DNA, $1 \mu \mathrm{L}$ of each primer $(50 \mathrm{pmol})$, and $38 \mu \mathrm{L}$ of distilled water in a final volume of $50 \mu \mathrm{L}$. The cycling profile was denaturation at $95^{\circ} \mathrm{C}$ for $5 \mathrm{~min}$ followed by 35 cycles of denaturation at $95^{\circ} \mathrm{C}$ for $30 \mathrm{sec}$, annealing at $55^{\circ} \mathrm{C}$ for $45 \mathrm{sec}$, and extension at $72^{\circ} \mathrm{C}$ for $1 \mathrm{~min}$, with a final extension at $72^{\circ} \mathrm{C}$ for $5 \mathrm{~min}$. The products were visualized by $1.8 \%$ agarose gel electrophoresis with RedSafe Nucleic Acid Staining Solution (iNtRON Biotechnology). Lung samples with a 757-bp product were considered positive. All brain and lung samples were inoculated into Vero cells (CRL1586; American Type Culture Collection [ATCC], USA) and MDCK cells (CCL34; ATCC) to isolate CHV. All samples that did not show specific cytopathic effects (CPEs) were passaged three times in the same cells.

\section{Detection of antibodies against CDV, CPV and CHV by ELISAs \\ ELISA kits for CDV (INgezim MOQUILLO IgG; Inmu-} nología y Genética Aplicada, Spain), CPV (INgezim; Inmunología y Genética Aplicada) and CHV (arigo Biolaboratories, Taiwan) were used according to the manufacturer's instructions to detect antibodies against $\mathrm{CDV}, \mathrm{CPV}$, and $\mathrm{CHV}$. In brief, the serum samples were diluted 1:100 (CDV and CPV) or 1:200 (CHV) and added to the ELISA kit. After $10 \mathrm{~min}$ of incubation at room temperature, the plates were washed four times with washing solution and then $100 \mu \mathrm{L}$ of conjugate was added to each well. The sealed plates were incubated for $10 \mathrm{~min}$ at room temperature, the conjugate was discarded, and the plates were washed four times. Finally, $100 \mu \mathrm{L}$ of substrate solution was added and the plates were kept for $5 \mathrm{~min}$ at room temperature. After adding 50 or $100 \mu \mathrm{L}$ of stop solution to each well, the absorbance of each well was read with an ELISA reader (Sunrise; Tecan, Switzerland) at $450 \mathrm{~nm}$. The results were interpreted based on positive or negative samples according to the instructions. The ELISA optical density values were equivalent to each viral neutralization (VN) titer.

\section{Virus neutralization of CAdV-2}

The CAdV-2 (strain QIAA1601) used in this study was isolated from a lung sample of naturally infected dogs in 2016. The CAdV-2 strain had been propagated in Vero cells and the CAdV-2 titer was confirmed by CPEs and an indirect fluorescent assay with anti-CAdV-2 antibodies (4H1-A7; VMRD, USA). CAdV-2 was used as the antigen in a VN test. The VN test was performed in 96-well plates in duplicate using sera inactivated at $56^{\circ} \mathrm{C}$ for $30 \mathrm{~min}$. Then, $50 \mu \mathrm{L}$ aliquots of two-fold serially diluted serum were mixed with equal volumes of CAdV-2 containing $200 \mathrm{TCID}_{50} / 0.1 \mathrm{~mL}$. After incubating the mixtures at $37^{\circ} \mathrm{C}$ for $1 \mathrm{~h}, 100 \mu \mathrm{L}$ of a Vero cell suspension containing 20,000 cells was added to each well. The plates were incubated for 3 days in a humidified incubator with $5 \% \mathrm{CO}_{2}$. Each well was examined under a microscope to detect viral-specific CPEs. The virus neutralizing antibody (VNA) titers were expressed as the reciprocal of the highest serum dilution that completely inhibited CPEs. Sera showing a VNA titer $\geq 1: 2$ were considered positive.

\section{Hemagglutination inhibition (HI) test for CPIV-5}

CPIV-5 (strain QIA-B1201) was used as the antigen for the HI test. The hemagglutination (HA) test was performed by 
preparing serial two-fold dilutions of CPIV-5 in $50 \mu \mathrm{L}$ of PBS (pH 7.2) and adding $50 \mu \mathrm{L}$ of $0.6 \%$ chicken erythrocytes. The HI test was performed in 96-well microplates as described previously $[1,23]$. The HI titer was expressed as the reciprocal of the highest dilution of serum showing complete inhibition of HA. Serum samples showing HI titers $\geq 1: 10$ were considered positive.

A
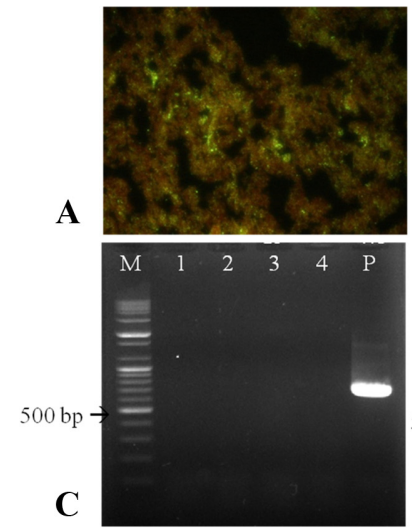

B
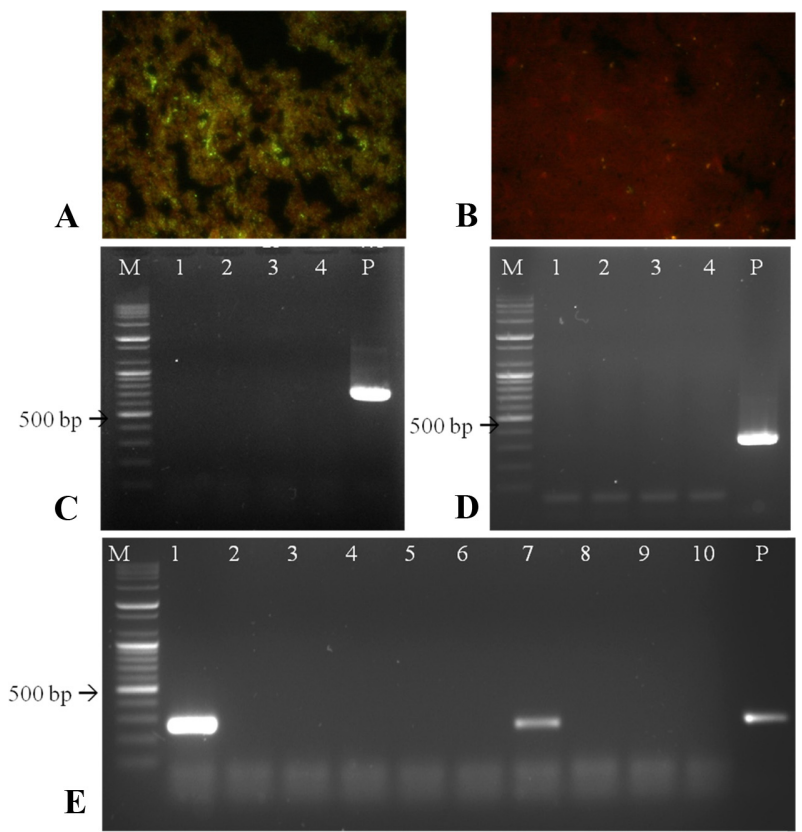

Fig. 1. Rabies virus (RABV) positive (A) and negative (B) samples by the direct fluorescent antibody test. There was no positive RABV reaction in the 62 raccoon dog brain samples. Attempts at detecting canine adenovirus type 2 (CAdV-2) (C) and canine parainfluenza virus type 5 (CPIV-5) (D) in lung samples. M, DNA ladder; Lanes 1-4, lung samples; P, positive sample. There were no positive samples against CAdV-2 or CPIV5. Detection of canine distemper virus (CDV) in Korean raccoon dog brain samples (E). Lanes 1-10, brain samples; P, positive sample. Samples 1 and 7 showed positive reactions against CDV.

$\mathbf{A}$

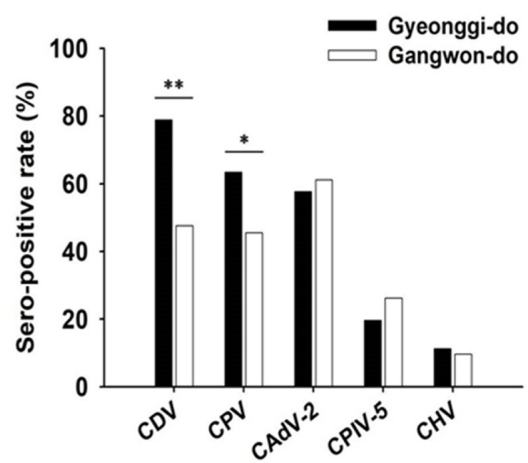

\section{Statistical analysis}

Chi-square test was used to compare the prevalence rate of each virus between the two different geographic regions. The statistical analysis was performed with SPSS statistics 21.0 software (IBM, USA). A $p$ value $<0.05$ was considered significant.

\section{Results}

In total, 62 brain and 49 lung samples were collected from raccoon dogs held at the Wild Animal Rescue Center of Kangwon National University, and RABV, CDV, CAdV-2, CPIV-5, and CHV antigens were detected. As shown in Figure 1 , no RABV antigens were detected by the DFA test in the 62 brain samples, and no CAdV-2 or CPIV-5 antigens were detected in the 49 lung samples by PCR or RT-PCR. However, nine CDV antigens $(8.1 \%, 9 / 111)$ in 62 brain and 49 lung samples were detected by RT-PCR. Six brain and three lung samples showed positive reactions against CDV. No specific CPEs were observed in Vero or MDCK cells inoculated with the 62 brain and 49 lung samples for the isolation of CHV.

The seroprevalence of five viruses was examined by ELISA, VN, and HI tests in 174 raccoon dog sera collected from Gyeonggi and Gangwon Provinces. As shown in Figure 2, the overall seropositive rates against CDV, CPV, CAdV-2, CPIV-5, and CHV were 60.3\% (105/174), 52.9\% (92/174), 59.8\% (104/174), 23.6\% (41/174), and 10.3\% (18/ 174), respectively. The regional incidence rates of $\mathrm{CDV}$, CPV, and CAdV-2 were 78.9\% (56/71), 63.4\% (45/71), and $57.7 \%(41 / 71)$ in Gyeonggi province and $47.0 \%$ (49/103), $45.6 \%$ (47/103), and 61.2\% (63/103) in Gangwon Province, respectively (Fig. 2A). The raccoon dogs captured from Gyeonggi Province showed higher incidence rates of CDV (78.9\%), CPV (63.4\%), and CHV (11.3\%) than those from Gangwon Province, and the raccoon dogs from Gangwon Province showed slightly higher incidence rates of CPIV-5 (26.2\%) and CAdV-2 (61.2\%) than those from Gyeonggi Province.

B

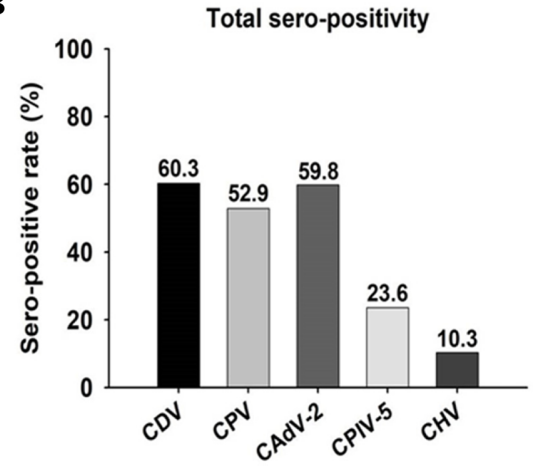

Fig. 2. Sero-positive rates against canine distemper virus (CDV), canine parvovirus (CPV), canine adenovirus type 2 (CAdV-2), canine parainfluenza virus type 5 (CPIV-5), and canine herpesvirus (CHV) in Korean raccoon dog sera. There were regional differences in the sero-positive rates against CDV and CPV (A). The sero-positive rate $(60.3 \%)$ of CDV was the highest of the five pathogens (B). ${ }^{*} p<0.001$ and ${ }^{* *} p<0.05$, respectively. 


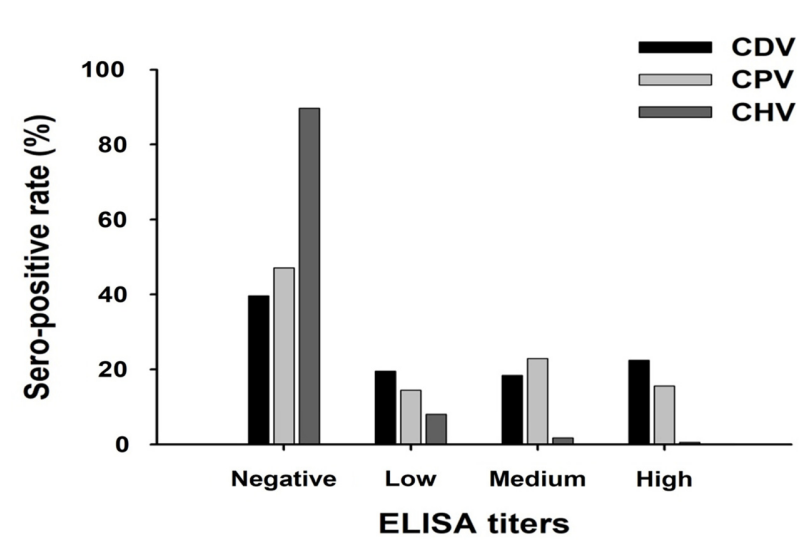

Fig. 3. Distribution of enzyme-linked immunosorbent assay titers against canine distemper virus (CDV), canine parvovirus (CPV), and canine herpesvirus (CHV) in Korean raccoon dog sera.

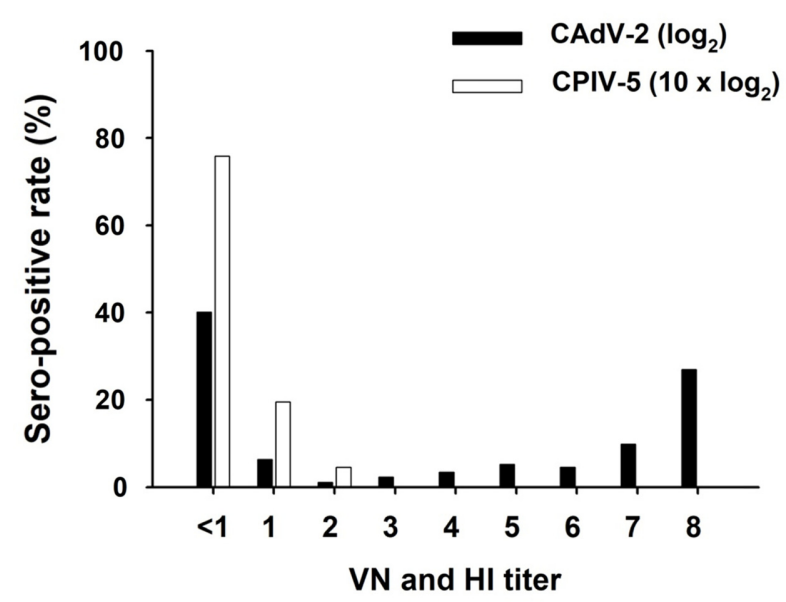

Fig. 4. Distribution of virus neutralizing (VN) antibody and hemagglutination inhibition (HI) titers against canine adenovirus-2 (CAdV-2) and canine parainfluenza virus type 5 (CPIV-5) in Korean raccoon dog sera.

Analyzing the distribution of ELISA titers against CDV, $\mathrm{CPV}$, and $\mathrm{CHV}$ showed that there were no significant differences in the low, medium, and high ELISA titers of CDV and CPV. However, the incidence rate of raccoon dogs representing a low ELISA titer against CHV was $8.1 \%$ (Fig. 3). As shown in Figure 4, in the raccoon dogs that had VNA titers $>\log _{2} 1: 2$ or $1: 10$ against CAdV-2 or CPIV-5, the most frequent VNA titers against CAdV-2 and CPIV-5 were $\log _{2} 2^{8}$ $(27.0 \%)$ and $10 \times \log _{2} 2^{1}(19.5 \%)$, respectively.

\section{Discussion}

The purpose of this study was to identify which viral antigens were detected from dead raccoon dogs and to determine if the raccoon dogs living in two provinces were exposed and had antibodies to any of the viruses. RABV, CPIV-5, and CAdV-2 were not detected by DFA, RT-PCR, or PCR, whereas nine CDV antigens were detected by RT-PCR in six brain and three lung samples. No CHV was isolated from Vero or MDCK cells inoculated with the above samples. The sero-positive rates of CDV, CPV, CAdV-2, CPIV-5, and $\mathrm{CHV}$ were $60.3 \%, 52.9 \%, 59.8 \%, 23.6 \%$, and $10.3 \%$, respectively.

A significant finding of this study is that no RABV antigen was detected in any of the 62 brain samples of raccoon dogs. Raccoon dog-mediated rabies in Korea was first identified in 1993, and the number of animal rabies cases continued to increase until 2002 when 78 rabies cases were confirmed and linked to rabid raccoon dogs [5]. The veterinary authority decided on a policy to distribute rabies bait vaccine and conduct sero-surveillance of dogs and cattle in 2002 to block the transmission of raccoon dog-mediated rabies. The doses of the bait vaccine reached 98 million in 2016 [21]. Although the reinforced national rabies control program has led to a meaningful reduction in animal rabies, continuous surveillance should be undertaken in suspected animals, including raccoon dogs, because of the possible recurrence of rabies in wild raccoon dogs, which are known to be a reservoir host.

One of the most frequent causes of death in raccoon dogs is CDV infection. Many cases of CDV infection have been identified in wild Korean raccoon dogs $[4,7]$. In this study, nine CDV antigens were detected in brain and lung samples, indicating the strong possible transmission of CDV from raccoon dogs to companion animals through accidental contact. This speculation is backed up by sero-surveillance results using raccoon dog sera. Korean raccoon dogs had a higher CDV sero-positive rate $(60.3 \%)$ compared to CPV (52.9\%), CAdV-2 (59.8\%), CPIV-5 (23.6\%), or CHV (10.3\%). Although there were slight regional differences in the sero-surveillance results, Korean raccoon dogs seemed to have high sero-positive against CDV, ranging from 44.1 to $89.4 \%$ [4, 22]. However, only $7 \%$ of Japanese raccoons are sero-positive for CDV, indicating geographical differences and a relatively low risk of transmission in Japan [2].

Moreover, CPV infections causing severe bloody diarrhea have been reported in dogs and wild raccoon dogs [8, 11, 12, 14]. In this study, $52.9 \%$ of Korean raccoon dogs had antiCPV antibodies, indicating that CPV infection is widespread in the wild raccoon dog population. This serological evidence of exposure to CPV suggests that raccoon dogs infected with CPV play an important role as a reservoir and that this pathogen has been spreading to other carnivores through unintentional contact.

CAdV-2 and CPIV-5 have been reported as causative viral agents of upper respiratory disease in dogs and red fox species in Europe [3, 19]. Animals infected with these viruses and Bordetella bronchiseptica simultaneously develop severe respiratory illness symptoms [17]. CPIV-5s were isolated from lung tissues of Korean and Chinese dogs with mild pneumonia $[15,16]$, but there are no reports of CAdV-2 and CPIV-5 infections in raccoon dogs in Korea. In this study, although the CAdV-2 antigen was not detected by PCR in lung samples, $59.8 \%$ of raccoon dogs had VNA against CAdV-2 and 
$27 \%$ of raccoon dogs had a high VNA titer $>1: 256$, indicating that many raccoon dogs have recently been infected and that these viruses are circulating in the population. Unlike CAdV-2, 23.6\% of raccoon dogs had the HI antibody against CPIV-5 and the level of the CPIV-5 antibody was $\leq 1: 20$, indicating that CPIV-5 infection is rare in the wild raccoon dog population. Bitches infected with CHV develop reproductive problems, including aborted, still-born, or weak puppies [10]. Serological evidence of CHV in dogs varies from 20 to $94 \%$ depending on the country [13]. In this study, only $10.3 \%$ of raccoon dogs had low antibody levels against CHV in the ELISA, suggesting that CHV infection is not severe in raccoon dogs.

In conclusion, with regard to the "One Health" concept, this study emphasizes the importance of raccoon dog-mediated rabies. Although the RABV antigen was not identified, our results reveal evidence of exposure to CDV, CPV, CAdV2, CPIV-5, and CHV in raccoon dogs. Accidental contact among humans, companion animals, and wild raccoon dogs can play a subordinate role in the transmission of infectious pathogens, including bacteria and external parasites. Therefore, continuous and periodic investigations into the health status of wild raccoon dogs is needed and further sero-surveillance of RABV in raccoon dogs captured from regions where the rabies bait vaccine has been distributed is required in the near future.

\section{Acknowledgments}

This work was supported financially by a grant (2016-269) from Korea Institute of Planning \& Evaluation for Technology (IPET), Ministry of Agriculture, Food and Rural Affairs (MAFRA), Republic of Korea.

\section{References}

1. Ajiki M, Takamura K, Hiramatsu K, Nakai M, Sasaki N, Konishi S. Isolation and characterization of parainfluenza 5 virus from a dog. Nihon Juigaku Zasshi 1982, 44, 607-618.

2. Aoki E, Soma T, Yokoyama M, Matsubayashi M, Sasai K. Surveillance for antibodies against six canine viruses in wild raccoons (Procyon lotor) in Japan. J Wildl Dis 2017, 53, 761-768.

3. Balboni A, Verin R, Morandi F, Poli A, Prosperi S, Battilani M. Molecular epidemiology of canine adenovirus type 1 and type 2 in free-ranging foxes (Vulpes vulpes) in Italy. Vet Microbiol 2013, 162, 551-557.

4. Cha SY, Kim EJ, Kang M, Jang SH, Lee HB, Jang HK. Epidemiology of canine distemper virus in wild raccoon dogs (Nyctereutes procyonoides) from South Korea. Comp Immunol Microbiol Infect Dis 2012, 35, 497-504.

5. Cheong Y, Kim B, Lee KJ, Park D, Kim S, Kim H, Park E, Lee H, Bae C, Oh C, Park SY, Song CS, Lee SW, Choi IS, Lee JB. Strategic model of national rabies control in Korea. Clin Exp Vaccine Res 2014, 3, 78-90.

6. Cho A, Roh YS, Lee HB, Cho HS, Lim CW, Kang SJ, Kim HY, Kim J, Kim B. Canine distemper virus infection in a wild Korean raccoon dog. J Prev Vet Med 2015, 39, 29-32.

7. Cho HS, Kang JI, Park NY. Detection of canine parvovirus in fecal samples using loop-mediated isothermal amplification. J Vet Diagn Invest 2006, 18, 81-84.

8. Frölich K, Streich WJ, Fickel J, Jung S, Truyen U, Hentschke J, Dedek J, Prager D, Latz N. Epizootiologic investigations of parvovirus infections in free-ranging carnivores from Germany. J Wildl Dis 2005, 41, 231-235.

9. Glynn MK, Brink N. Perspectives on One Health: a survey of national Delegates to the World Organisation for Animal Health, 2012. Rev Sci Tech 2014, 33, 433-441.

10. Hashimoto A, Hirai K, Suzuki Y, Fujimoto Y. Experimental transplacental transmission of canine herpesvirus in pregnant bitches during the second trimester of gestation. Am J Vet Res 1983, 44, 610-614.

11. Kang BK, Song DS, Lee CS, Jung KI, Park SJ, Kim EM, Park BK. Prevalence and genetic characterization of canine parvoviruses in Korea. Virus Genes 2008, 36, 127133.

12. Kapil S, Rezabek G, Germany B, Johnston L. Isolation of a virus related to canine parvovirus type 2 from a raccoon (Procyon lotor). Vet Rec 2010, 166, 24-25.

13. Krogenæes A, Rootwelt V, Larsen S, Renström L, Farstad W, Lund A. A serological study of canine herpesvirus-1 infection in a population of breeding bitches in Norway. Acta Vet Scand 2014, 56, 19.

14. Litster A, Nichols J, Volpe A. Prevalence of positive antibody test results for canine parvovirus (CPV) and canine distemper virus (CDV) and response to modified live vaccination against CPV and CDV in dogs entering animal shelters. Vet Microbiol 2012, 157, 86-90.

15. Liu C, Li X, Zhang J, Yang L, Li F, Deng J, Tan F, Sun M, Liu Y, Tian K. Isolation and genomic characterization of a canine parainfluenza virus type 5 strain in China. Arch Virol 2017, 162, 2337-2344.

16. Oem JK, Kim SH, Kim YH, Lee MH, Lee KK. Molecular characteristics of canine parainfluenza viruses type 5 (CPIV5) isolated in Korea. Can J Vet Res 2015, 79, 64-67.

17. Quan SF, Witten ML, Dambro NN, Lemen RJ. Canine parainfluneza type 2 and Bordetella bronchiseptica infection produces increased bronchoalveolar lavage thromboxane concentrations in Beagle puppies. Prostaglandins Leukot Essent Fatty Acids 1991, 44, 171-175.

18. Rentería-Solís Z, Förster C, Aue A, Wittstatt U, Wibbelt G, König M. Canine distemper outbreak in raccoons suggests pathogen interspecies transmission amongst alien and native carnivores in urban areas from Germany. Vet Microbiol 2014, 174, 50-59.

19. Robinson AJ, Crerar SK, Waight Sharma N, Müller WJ, Bradley MP. Prevalence of serum antibodies to canine adenovirus and canine herpesvirus in the European red fox (Vulpes vulpes) in Australia. Aust Vet J 2005, 83, 356-61.

20. World Organization for Animal Health (OIE). Manual of Diagnostic Tests and Vaccines for Terrestrial Animals. 7th ed. pp. 263-282, OIE, Paris, 2012.

21. Yang DK, Kim HH, Lee KK, Yoo JY, Seomun H, Cho IS. Mass vaccination has led to the elimination of rabies since 2014 in South Korea. Clin Exp Vaccine Res 2017, 6, 111-119. 
22. Yang DK, Kim HH, Nah JJ, Choi SS, Kim JT, Jeong WH, Song JY. Serologic survey of rabies virus, canine distemper virus and parvovirus in wild raccoon dogs (Nyctereutes procyonoides koreensis) in Korea. J Bacteriol Virol 2013, 43, 204-209.
23. Yang DK, Nah JJ, Kim HH, Choi SS, Bae YC, Park JW, Song JY. Isolation of novel parainfluenza virus type 5 (bPIV5) and its incidence in Korean cattle. Korean J Vet Res 2014, 54, 107-112. 